\title{
Role of Primers in Glucan Synthesis by Glucosyltransferases from Streptococcus mutans strain OMZ176
}

\author{
By TOSHIHIKO KOGA, ${ }^{1,2}$ SETSUKO SATO,${ }^{1}$ MASAKAZU INOUE, ${ }^{1 *}$ \\ KANO TAKEUCHI, ${ }^{2}$ TAKUSHI FURUTA ${ }^{2}$ AND SHIGEYUKI HAMADA ${ }^{2}$ \\ ${ }^{1}$ Department of Preventive Dentistry, Kagoshima University Dental School, 1208-1, Usuki-cho, \\ Kagoshima 890, Japan \\ ${ }^{2}$ Department of Dental Research, National Institute of Health, Kamiosaki, Shinagawa-ku, \\ Tokyo 141, Japan
}

(Received 12 July 1982; revised 28 October 1982)

\begin{abstract}
The effects of isomaltosaccharides of various molecular weights (isomaltose to dextran T2000) on glucan synthesis by a water-soluble glucan-synthesizing glucosyltransferase enzyme (GTaseS) and a water-insoluble glucan-synthesizing enzyme (GTase-I), both from Streptococcus mutans OMZ176, were examined. The activity of GTase-S was not affected by the addition of the isomaltosaccharides, but GTase-I was stimulated increasingly by isomaltosaccharides with degrees of polymerization more than 10 . The GTase-I activity first increased and thereafter decreased slightly with increasing amounts of a soluble dextran. Maximal stimulation occurred at concentrations in the range 0.1 to $0.2 \mathrm{mg} \mathrm{ml}^{-1}$, when dextran T10 was used as a primer. The rate of glucan synthesis was highly enhanced by the combined action of GTase-S and GTase-I. The profile of the net activity of GTase-I in the presence of various amounts of GTase-S was similar to that of GTase-I in the presence of increasing amounts of an exogenous dextran. These results collectively suggest that soluble glucan produced by GTase-S from sucrose acts as an intrinsic primer for the glucan synthesis by GTase-I, indicating the contribution of autopriming in glucan synthesis by crude GTase of $S$. mutans.
\end{abstract}

\section{INTRODUCTION}

Streptococcus mutans is a prime etiological agent of human dental caries (Gibbons \& van Houte, 1975; Hamada \& Slade, 1980). Water-insoluble and water-soluble glucans produced from sucrose by $S$. mutans play a crucial role in the formation and metabolism of dental plaque and consequently in the induction of dental caries (Hamada \& Slade, 1980). These glucans are synthesized by the action of glucosyltransferases (GTase; EC 2.4.1.5).

Enzymic activity of $S$. mutans GTases is stimulated by the addition or presence of exogenous dextrans (primer effect) (Germaine et al., 1974a; Kuramitsu, 1975; Germaine \& Schachtele, 1976; Newbrun et al., 1977; Robyt \& Corrigan, 1977; Hare et al., 1978; McCabe \& Smith, 1978; Hamada \& Torii, 1980; Sato et al., 1982). The degree of stimulation varies with different GTase preparations from a single strain of $S$. mutans, but the mechanism is unknown.

Recently, we reported the separation of a water-soluble glucan-synthesizing component (GTase-S) and a water-insoluble glucan-synthesizing component (GTase-I) from crude GTase obtained from culture supernatant of a serotype $d$ strain of $S$. mutans (Koga et al., 1982). We now describe the effects of various isomaltosaccharides on glucan synthesis by these purified GTase preparations and discuss the role of primers in the synthesis of water-insoluble glucan by $S$. mutans GTase.

\section{METHODS}

GTase preparations. GTase-S and GTase-I were separated by the chromatofocusing method and subsequent hydroxyapatite column chromatography from crude GTase obtained by $50 \%$ ammonium sulphate precipitation of 
cell free culture supernatants of Streptococcus mutans OMZ176 (serotype $d$ ) as described previously (Koga et al., 1982). Specific enzyme activities of the separated GTase-S and GTase-I were 3.7 and $1.8 \mathrm{U}$ (mg protein) ${ }^{-1}$, respectively. One unit was defined as the amount of enzyme that transformed $1 \mu \mathrm{mol}$ sucrose into glucan per min, under the conditions described previously (Koga et al., 1982).

Assay for GTase activity. Glucan-synthesizing activity of the GTases was measured by a modification of the method described by Germaine et al. $(1974 b)$. The standard reaction mixture contained appropriate amounts $(0$ to $0.37 \mathrm{mU})$ of GTase and $0.04 \mu \mathrm{Ci}$ of $\left[\mathrm{U}^{-14} \mathrm{C}\right]$ sucrose $\left(0.2 \mathrm{Ci} \mathrm{mol}^{-1}, 7.4 \mathrm{GBq}\right.$; New England Nuclear $)$ in a total volume of $20 \mu \mathrm{l} 0 \cdot 1 \mathrm{M}$-potassium phosphate buffer $\left(\mathrm{pH} \mathrm{6.0)}\right.$. After the incubation for 0 to $2 \mathrm{~h}$ at $37^{\circ} \mathrm{C}$, the amount of $\left[{ }^{14} \mathrm{C}\right]$ glucose polymerized was estimated by the filter paper method (Koga \& Inoue, 1981).

To examine the effects of isomaltosaccharides on glucan synthesis by the GTase components, 0 to $0.2 \mathrm{mg}$ of the saccharides were added to the above mixture and incubated.

Isomaltosaccharides. Dextran T10 (mean molecular weight 10000), T20 (20000), T40 (40000), T250 (250000), T500 (500000) and T2000 (2000000) were obtained from Pharmacia.

Preparation of oligosaccharides of the isomaltose series. Dextran $\mathrm{T} 40\left(0 \cdot 1 \mathrm{~g} \mathrm{ml}^{-1}\right)$ was partially hydrolysed in

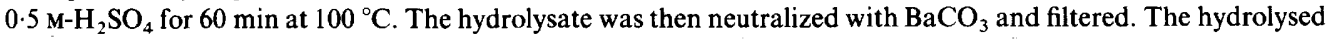
products were precipitated from the filtrate by the addition of cold absolute ethanol to a final concentration of $80 \%$ $(v / v)$, collected by centrifugation, and solubilized in a small volume of water. Oligosaccharides with various degrees of polymerization (DP) were separated by paper chromatography and subsequent column chromatography on Bio-Gel P2 (Bio-Rad). The DP was determined by calculating molar proportions of total hexose residues to reducing sugar residues.

Chemical assays. Protein was estimated by the Lowry method with bovine serum albumin as a standard. Reducing sugar and total hexose were measured by the method of Somogyi-Nelson (Nelson, 1944) and by the anthrone method (Trevelyan \& Harrison, 1956), respectively, with glucose as a standard.

\section{RESULTS}

\section{Effects of isomaltosaccharides on glucan synthesis by GTase-S and GTase-I}

GTase-I ( $0.24 \mathrm{mU})$ had synthesized only trace amounts of glucan from sucrose in the absence of dextran T10 even after $2 \mathrm{~h}$ incubation, but the presence of the soluble dextran at a $0 \cdot 2 \mathrm{mg} \mathrm{ml}^{-1}$ concentration significantly stimulated glucan synthesis by GTase-I.

The degree of stimulation of the rate of glucan synthesis increased with increasing amounts of the exogenous dextran T10 up to $0.2 \mathrm{mg} \mathrm{ml}^{-1}$ but decreased at dextran concentrations above $0.2 \mathrm{mg} \mathrm{ml}^{-1}$ (Fig. $1 a$ ). Significant stimulation of glucan synthesis was caused when dextran was included at concentrations of 0.1 to $0.2 \mathrm{mg} \mathrm{ml}^{-1}$. The extent of the maximum stimulation of glucan synthesis was approximately 15 -fold.

The ability of a constant amount, $0.2 \mathrm{mg} \mathrm{ml}^{-1}$, of isomaltosaccharides with various DP to prime GTase-I activity was assessed. Glucan synthesis by GTase-I was stimulated by isomaltosaccharides with DP higher than 10, the extent of stimulation increasing with DP. However, the isomaltooligosaccharides with DP of less than 8 were essentially without effect (Fig. $1 b)$. In contrast, glucan synthesis by GTase-S $(0 \cdot 37 \mathrm{mU})$ was not influenced by the addition of dextran $\mathrm{T} 10$ at concentrations in the range 0.01 to $10 \mathrm{mg} \mathrm{ml}^{-1}$ or by $0.2 \mathrm{mg} \mathrm{m}^{-1}$ of isomaltosaccharides (Fig. 1).

\section{Role of primers in the glucan synthesis by the combined action of the isolated glucosyltransferase components}

When a constant amount of GTase-I $(0.24 \mathrm{mU})$ and varying amounts of GTase-S (0 to $0.074 \mathrm{mU}$ ) were incubated with $10 \mathrm{~mm}$-sucrose, the quantities of glucan synthesized by the combined action of the two GTase components were far higher than the additive amounts of glucan produced by these GTases separately (Fig. 1c). The addition of $0.2 \mathrm{mg}$ exogenous dextran $\mathrm{T} 10 \mathrm{ml}^{-1}$ stimulated glucan synthesis by the combined action of the GTase components when the activity ratio of GTase-S to GTase-I was less than 0.08 (Fig. 1c). However, if the ratio was more than 0.08 , glucan synthesis was slightly inhibited. These results were repeatedly demonstrated. Thus, the stimulatory or inhibitory effects of exogenous soluble dextrans were strongly dependent upon the relative proportions of GTase-S and GTase-I. The maximum stimulation occurred when the ratio of GTase-S to GTase-I activities was approximately 0.15 

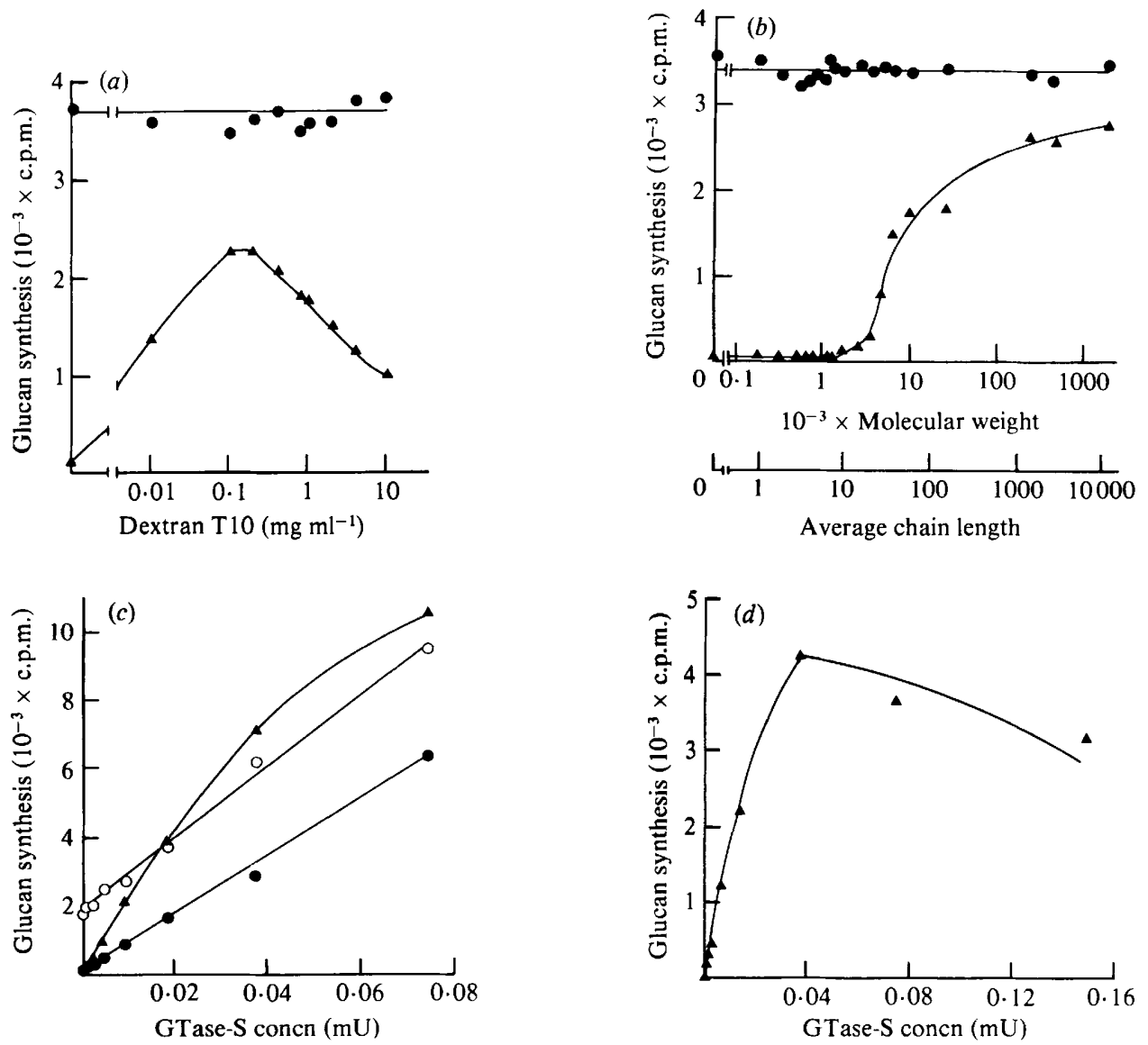

Fig. 1(a,b). Effect of dextran T10 (a) and various isomaltosaccharides $(b)$ on glucan synthesis, measured as the uptake of $\left[\mathrm{U}-{ }^{14} \mathrm{C}\right]$ sucrose, by GTase-S $(\boldsymbol{O})$ and GTase-I (A). (a) GTase-S $(0 \cdot 37 \mathrm{mU})$ or GTase-I $(0.24 \mathrm{mU})$ was incubated with [U-14 $\mathrm{C}$ ]sucrose $(0.2 \mu \mathrm{mol}, 0.04 \mu \mathrm{Ci})$ in the presence of various amounts of dextran T10. After $60 \mathrm{~min}$ at $37^{\circ} \mathrm{C}$, the amounts of $\left[{ }^{14} \mathrm{C}\right]$ glucan synthesized were estimated by the filter paper method. (b) Experimental conditions were identical to those described for $(a)$ except that dextran T10 was replaced by isomaltosaccharides $(4 \mu \mathrm{g})$. (c) Glucan synthesis by the combined action of GTase-S and GTase-I. GTase-I (0.24 mU) and various amounts (0 to 0.074 mU) of GTase-S were incubated together at $37^{\circ} \mathrm{C}$ for $60 \mathrm{~min}$ with [ $\mathrm{U}^{-14} \mathrm{C}$ ]sucrose $(0.2 \mu \mathrm{mol}, 0.04 \mu \mathrm{Ci})$ in the absence $(\triangle)$ or presence $(\mathrm{O})$ of dextran T10 $(4 \mu \mathrm{g})$. The reaction mixture without GTase-I and dextran T10 served as controls (O). (d) Stimulation of GTase-I-dependent glucan synthesis by GTase-S. The data are taken from the experiment described in $(c)$. The net amounts of glucan produced by GTase-I were obtained by subtracting the amounts of glucan synthesized by GTase-S alone from those of glucan synthesized by GTase-I and GTase-S together but in the absence of dextran T10.

(Fig. $1 d$ ). The profile shown in Fig. $1(d)$ is similar to that obtained with the stimulation of GTase-I activity by an exogenous soluble dextran (Fig. 1a).

\section{DISCUSSION}

Stimulation of the $S$. mutans GTase-I activity by oligo- and polysaccharides of the isomaltose series has been demonstrated for the extracellular enzyme of $S$. mutans GS-5 (serotype $c$ ) and OMZ176 (serotype $d$ ) (Germaine et al., 1974a; Hare et al., 1978). Germaine et al. (1974a) have reported that the smallest effective primer is the isomaltosaccharide with DP of about 8 , whereas our study indicates that primer activity of isomaltosaccharides commenced with DP of 10 or more (Fig. $1 b$ ). The difference in the minimum DP for primer activity may be ascribed to 
the purity of isomaltosaccharides used. Our preparations were purified by gel filtration, followed by repeated separation of individual isomaltosaccharides by paper chromatography.

The GTase-S activity was not influenced by the addition of isomaltosaccharides tested (Fig. $1 a, b)$. Germaine et al. (1974a) reported that the extent of stimulation of GTase activity by primer dextran decreased with increasing amounts of carbohydrate in the GTase preparations used. However, the GTase-S preparation used in the present study has been shown to be carbohydrate free (Koga et al., 1982), indicating that the activity of the GTase-S of $S$. mutans OMZ176 (serotype $d$ ) is essentially primer independent.

As GTase-S activity was not stimulated by exogenous dextrans (Fig. $1 a, b$ ), the enhanced synthesis of glucan by the concerted action of the two GTase components (Fig. 1c) may be ascribed to the stimulation of GTase-I activity by glucan rich in 1,6- $\alpha$ linkages first synthesized by GTase-S (autopriming effect). The variation of glucan synthesis by GTase-I depended on the amounts of GTase-S included in the mixture (Fig. $1 d$ ), showing a profile similar to that of glucan synthesis by GTase-I in the presence of exogenous dextran (Fig. 1a). This supports the suggestion that autopriming occurs during insoluble glucan synthesis by crude $S$. mutans GTases (Germaine et al., 1977; Kuramitsu, 1975).

\section{REFERENCES}

Germaine, G. R. \& Schachtele, C. F. (1976). Streptococcus mutans dextransucrase: mode of interaction with high-molecular-weight dextran and role in cell aggregation. Infection and Immunity 13, 365-372.

Germaine, G. R., Chludzinski, A. M. \& SchachTELE, C. F. (1974a). Streptococcus mutans dextransucrase: requirement for primer dextran. Journal of Bacteriology 120, 287-294.

Germaine, G. R., SCHACHTEle, C. F. \& Chludzinski, A. M. (1974b). Rapid filter paper assay for the dextransucrase activity from Streptococcus mutans. Journal of Dental Research 53, 1355-1360.

Germaine, G. R., Harlander, S. K., LeUng, W.-L. S. \& SChaChtele, C. F. (1977). Streptococcus mutans dextransucrase: functioning of primer dextran and endogenous dextranase in water-soluble and waterinsoluble glucan synthesis. Infection and Immunity 16, 637-648.

GibBons, R. J. \& VAN Houte, J. (1975). Dental caries. Annual Review of Medicine 29, 19-44.

Hamada, S. \& Slade, S. (1980). Biology, immunology, and cariogenicity of Streptococcus mutans. Microbiological Review 44, 331-384.

HAMADA, S. \& TORII, M. (1980). Interaction of glucosyltransferase from Streptococcus mutans with various glucans. Journal of General Microbiology 116, 51-59.

Hare, M. D., Svensson, S. \& Walker, G. J. (1978). Characterization of the extracellular, water-insoluble $\alpha$-D-glucans of oral streptococci by methylation analysis, and by enzymic synthesis and degradation. Carbohydrate Research 66, 245-264.

KOGA, T. \& INOUE, M. (1981). Inactivation of Dglucosyltransferases from oral Streptococcus mutans and Streptococcus sanguis by photochemical oxidation. Carbohydrate Research 93, 125-133.

Koga, T., SATo, S., YakushiJi, T. \& INoue, M. (1982). Separation of insoluble and soluble glucan-synthesizing glucosyltransferases of Streptococcus mutans. FEMS Microbiology Letters (in the Press).

KURAMITSU, H. K. (1975). Characterization of extracellular glucosyltransferase activity of Streptococcus mutans. Infection and Immunity 12, 738-749.

MCCABE, M. M. \& SMITH, E. E. (1978). The dextran acceptor reaction of dextransucrase from Streptococcus mutans K1-R. Carbohydrate Research 63, 223239.

Nelson, N. (1944). A photometric adaptation of the Somogyi method for the determination of glucose. Journal of Biological Chemistry 153, 375-380.

Newbrun, E., Finzen, F. \& Sharma, M. (1977). Inhibition of adherence of Streptococcus mutans to glass surfaces. Caries Research 11, 153-159.

RobYT, J. F. \& Corrigan, A. J. (1977). The mechanism of dextransucrase action. Activation of dextransucrase from Streptococcus mutans OMZ176 by dextran and modified dextran and the nonexistence of the primer requirement for the synthesis of dextran. Archives of Biochemistry and Biophysics 183, 726-731.

Sato, S., Koga, T., Yakushij, T., Nagasawa, S. \& INOUE, M. (1982). Effects of exogenous soluble dextrans on the insoluble glucan synthesis by Streptococcus mutans glucosyltransferase. Microbios 34, 99-112.

Trevelyan, W. E. \& Harrison, J. S. (1956). Studies on yeast metabolism. Biochemical Journal 63, 23-33. 